\section{Neuroscience from A to Z}

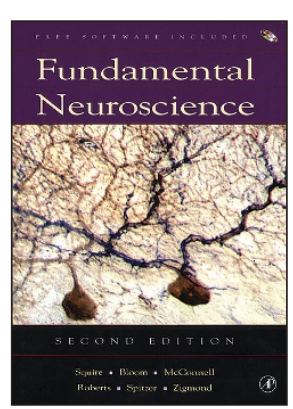

\section{Fundamental Neuroscience, Second Edition}

Edited by Larry R Squire, Floyd E Bloom, Susan K McConnell, James L Roberts, Nicholas C Spitzer \& Michael J Zigmond

Academic Press, 2003

1426 pp. hardcover, $\$ 119.95$

ISBN 0126603030

\section{Reviewed by Yehezkel Ben-Ari}

There are two categories of textbooks: those that attempt to describe in depth a restricted topic, and those that pursue a more general aim of reviewing an entire field. Fundamental Neuroscience belongs to the latter category. In the first edition of this textbook, the authors had the admirable, but formidable objective of presenting the full range of neuroscience. The second edition follows in the footsteps of its predecessor with similarly ambitious goals, continuing its coverage of topics ranging from the explosive advances of molecular and genetic approaches to imaging techniques in humans and the evolution of learning and behavior.

Summarizing a field as large as neuroscience to students and teachers is no easy task. To facilitate this goal, the authors provide abundant, well-designed illustrations to make complex mechanisms more comprehensible. Teachers will also find the multi-colored schematics useful for their courses. In a further effort to aid scanning such a large quantity of information, Fundamental Neuroscience includes boxes covering historical perspectives ("Cajal from iconoclast to icon"), medical diseases (HIV-associated dementia complex), presentations of techniques and preparations (the quail graft preparation in embryology) or summaries of major subjects (critical periods in humans or mechanisms of CNS synapse elimination). A well-organized index and excellent end-of-chapter summaries enable readers to rapidly find desired information.

The editors have selected some of the best experts in the various fields with contributions from over 130 authors, many of whom contributed to the first edition. The chapters dealing with brain development are particularly attractive, possibly because the advances in these fields - most notably in the identification of trophic and guiding factors - have led to an efficient and intelligible convergence of molecular and integrated studies. The debate on nature and nurture and the role of activity during construction of a cortical circuit are well explained, with elements taken from various preparations to

Yehezkel Ben-Ari is at the Institut de Neurobiologie de la Mediterranee (INMED) - INSERM U29, Parc Scientifique de Luminy, Marseilles 13273,

France.

email: ben-ari@inmed.univ-mrs.fr reconstruct the common features conserved throughout evolution. The result is a smooth marriage of genetic and functional studies and an efficient discussion of converging evidence available from different sources. For those interested in developmental neurobiology, the elegant chronological description of developmental sequences of molecular messages and intracellular cascades is highly recommended, although a more complete image of the field would require additional information on the electrical properties and firing patterns present in immature neurons.

Other chapters of Fundamental Neuroscience are less convincing and not up-to-date. For example, the LTP chapter includes a few mistakes. Metabotropic glutamate receptors are not essential for LTP induction but merely modulate LTP threshold by regulating NMDA receptor activity. There are also several inaccuracies concerning the locus of LTP expression. No reference is made to the fundamental topics of imaging techniques and gamma oscillations, which are providing new insight into plasticity. The contribution of GABAergic synapses to synaptic plasticity is also missing.

The chapters on neurotransmission provide another example of the difficulties in providing a clear summary of a complex field with several authors covering the topic. Classical neurotransmitters are introduced separately from explanations of the mechanism of transmitter release, neurotransmitter receptors and their molecular organization and the intracellular signaling or postsynaptic currents that result from transmitter action. This leads to a fragmented view of the topic. A chapter dealing with individual neurotransmitters (such as cholinergic transmission) that included molecular biology, receptor subunit composition, pharmacology, properties of synaptic currents and functional relevance in the CNS would be preferable. Such an organization would make the concepts and common rules that operate in synaptic transmission more comprehensible for neurobiology students. These problems are unavoidable in multi-author books, and this otherwise excellent textbook offers no solution to the problem. The mixture of styles and chapter organization is fortunately limited in Fundamental Neuroscience, most likely because of efficient work by the six editors.

Comparison with textbooks written by a few authors can also illustrate the advantages and limitations of multi-authored books like Fundamental Neuroscience. For example, Exploring the Brain by Bear, Connors and Paradiso (Lippincott Williams \& Wilkins, 2001) also aims to present the whole field of neuroscience with beautiful illustrations and diagrams, but is clearly targeted to a less-advanced audience. On the other hand, the primarily single-authored, specialized textbook Cellular and Molecular Neurobiology by Hammond ( $2^{\text {nd }}$ edition, Academic Press, 2001) illustrates an alternative way of teaching based on the use of classical experiments and a hypothesis-driven approach. The operation of synapses and receptors with all the complex biophysical and molecular machinery is organized in a functionoriented manner, providing the student and teacher with the unique opportunity to work in an enjoyable manner. There are extraordinary benefits to such participatory learning, in which one is involved in the thought processes leading to formulation of experiments and interpretation of results. Learning is both deeper and more enjoyable, and Hammond succeeds well at this ambitious task. 